Open Access

\title{
Mixed-mode growth of a multicomponent precipitate in the quasi-steady state regime
}

\author{
Tohid Naseri ${ }^{1}$, Daniel Larouche ${ }^{1 *} \mathbb{D}$, Rémi Martinez ${ }^{2}$ and Francis Breton ${ }^{3}$
}

\author{
* Correspondence: daniel.larouche@ \\ gmn.ulaval.ca \\ 'Department of Mining, Metallurgy \\ and Materials Engineering, \\ Aluminum Research Center - \\ REGAL, Laval University, 1065, Ave \\ de la Médecine, Quebec G1V 0A6, \\ Canada \\ Full list of author information is \\ available at the end of the article
}

\begin{abstract}
An exact analytical solution of the Fick's second law was developed and applied to the mixed-mode growth of a multicomponent ellipsoidal precipitate growing with constant eccentricities in the quasi-stationary regime. The solution is exact if the nominal composition, equilibrium concentrations and material properties are assumed constant, and can be applied to compounds having no limitations in the number of components. The solution was compared to the solution calculated by a diffusion-controlled application software and it was found that the solute concentrations at the interface can be determined knowing only the nominal composition, the full equilibrium concentrations and the coefficients of diffusion. The thermodynamic calculations owing to find alternative tie-lines are proven to be useless in the mixed-mode model. From this, it appears that the search of alternative tie-lines is computationally counterproductive, even when the interface has a very high mobility. A more efficient computational scheme is possible by considering that a moving interface is not at equilibrium.
\end{abstract}

Keywords: Mixed-mode, Interfacial mobility, Ellipsoidal precipitate, Multicomponent diffusion

\section{Introduction}

The diffusional growth of precipitates in a supersaturated matrix is one of the most fundamental problems in metallurgy. Although this topic is fully addressed in textbooks (Christian, 1965; Aaronson et al., 2010; Kozeschnik, 2013a), accurate estimations of growth velocities are difficult because of the assumptions considered to obtain a solution. For the time being, as pointed out by E. Kozeschnik in his book, "there exist no general closed analytical solutions for the multicomponent growth of precipitates" (Kozeschnik, 2013b). The only solutions available today are those obtained for binary systems in the quasi steady-state regime. Zener (Zener, 1949) provided the solution for spherical precipitates in the diffusion control mode while Horvey and Cahn (Horvay \& Cahn, 1961), as well as Ham (Ham, 1959), provided the solution in the same mode for the shape preserving growth of ellipsoidal precipitates. Larouche (Larouche, 2017) upgraded the solution of the latter precipitates for the mixed-mode growth regime, where the growth is partly controlled by diffusion and the interface. The reason why it is difficult to obtain a closed analytical solution for multicomponent precipitates when one assumes local equilibrium at the interface is because there are too many constraints at the interface. To understand the problem, let us consider the formation of a

(c) The Author(s). 2018 Open Access This article is distributed under the terms of the Creative Commons Attribution 4.0 International License (http://creativecommons.org/licenses/by/4.0/), which permits unrestricted use, distribution, and reproduction in any medium, provided you give appropriate credit to the original author(s) and the source, provide a link to the Creative Commons license, and indicate if changes were made. 
precipitate $A_{x} B_{y}$ in a binary system rich in species $A$. In the quasi steady-state regime, the boundary condition at infinity is $c=\bar{c}$ where $c$ is the molar concentration and $\bar{c}$ is the nominal composition of solute. The problem is solved by imposing two boundary conditions at the interface. The first one is the Stefan condition, which ensures that the velocity of the boundary is equal to the velocity imposed by the flux of atoms coming from the matrix. The second boundary condition is the concentration of solute at the interface, which is imposed by the local equilibrium assumption in the diffusion controlled mode. These two boundary conditions are enough to find the two unknowns at a given time, which are the size of the precipitate and the matrix solute concentration gradient at the interface. Problems arise when one considers the growth of a precipitate $\mathrm{B}_{\mathrm{x}} \mathrm{C}_{\mathrm{y}}$ in a ternary system rich in species $\mathrm{A}$. In this case, one unknown is added, which is the matrix solute concentration gradient at the interface of solute C. However, this adds two new boundary conditions. These are the Stefan boundary condition and the local equilibrium assumption for solute C. So, in this case, one has to deal with a problem having 4 boundary conditions at the interface and 3 unknowns, which are the size of the precipitate and the matrix solute concentration gradients at the interface for solutes $\mathrm{B}$ and $\mathrm{C}$. And for each species added in the composition of the precipitate, one has to add two new boundary conditions for only one extra unknown. To address this problem, Kirkaldy (Kirkaldy, 1958) and others suggested that there is only one independent solute concentration at the interface while the others are dependent to each other. Their determination depends on the operative tie-line in the phase diagram, which evolves according to the solute diffusivity and the degree of advancement of the reaction. In general, the operative tie-line differs from the conventional tie-line (determined when the system is at equilibrium) and the computational scheme used to solve the set of equations is not straightforward. Moreover, a mapping of the multicomponent phase diagram is mandatory to calculate the operative solute partitioning, even under isothermal growth. The application software DICTRA (Andersson et al., 2002) is well known to provide a numerical procedure solving the moving boundary problem for one-dimensional geometries. But one can testify about the difficulty of getting a solution with this software when the starting values cannot be found at the first time step. And this happens notably when the precipitate grows by the diffusion of two and more solute elements.

In this paper, an exact analytical solution of the shape preserving growth of a multicomponent ellipsoidal precipitate in the quasi stationary regime is presented. The solution is then applied for the growth of a $\beta$ " needle shaped particle growing in the $\mathrm{Al}-\mathrm{Mg}$-Si system. The advantages of this approach, involving the interfacial mobility, are further highlighted and discussed. Finally, a comparison with the solution provided by the application software DICTRA is performed for a spherical $\mathrm{Mg}_{2} \mathrm{Si}$ precipitate growing in an Aluminium matrix.

\section{The role of the interfacial mobility in the early stage of the growth}

When a precipitate $\beta$ is growing at the expense of a matrix $\alpha$, there is a flux $J_{1}\left(\mathrm{~mol} \mathrm{~m}^{-}\right.$ ${ }^{2} \mathrm{~s}^{-1}$ ) of atoms crossing the boundary in the direction of the precipitate and a flux $J_{2}$ of atoms going back to the matrix. The net rate of attachment is equal to $J_{1}-J_{2}$ multiplied by the surface of the boundary. If $\Delta \mu_{\mathrm{i}}\left(\mathrm{J} \mathrm{mol}^{-1}\right.$ of $\left.i\right)$ is the reduction of energy per 
mole of solute $i$, which remains attached to the precipitate, then the net reduction of free energy produced by the transfer of all species composing the precipitate will be:

$$
\Delta F^{*}=\sum_{i=1}^{I} N_{i} \Delta \mu_{i}
$$

Where $N_{i}$ is the number of moles of atoms condensing onto the precipitate and $I$ is the number of species composing the precipitate. Dividing Eq. (1) by the total number of moles of atoms captured by the precipitate one obtains (Hillert, 2008):

$$
\frac{d F^{*}}{d N}=\sum_{i=1}^{I} c_{\beta}^{i} \cdot \Delta \mu_{i}=\sum_{i=1}^{I} c_{\beta}^{i} \cdot\left(\mu_{i}^{\alpha *}-\mu_{i}^{\beta *}\right)
$$

Where $c_{\beta}^{i}$ is the molar fraction of element $i$ in the precipitate and $\mu_{i}^{\phi *}$ is the chemical potential of element $i$ in phase $\phi$ at the interface. The term $d F^{*} / d N$ is the driving force for boundary migration. For an ellipsoidal precipitate growing with constant eccentricities (See Fig. 1), one can characterize the boundary velocity by one of the principal directions.

Choosing the longest dimension $a_{1}(\mathrm{~m})$ to characterize the growth velocity of the precipitate, one can write (Christian, 1965):

$$
\frac{d a_{1}}{d t}=\frac{M}{V_{m}} \cdot \frac{d F^{*}}{d N}
$$

where $M\left(\mathrm{~m}^{4} \mathrm{~J}^{-1} \mathrm{~s}^{-1}\right)$ is the mobility of the interface and $V_{m}\left(\mathrm{~m}^{3} \mathrm{~mol}^{-1}\right)$ is the molar volume of the precipitate. Eq. (3) can be considered as the definition of the interfacial mobility, which in this case is related to the growth in the direction of the longest axis. One can evaluate the driving force with the following equation (Larouche, 2017):

$$
\frac{d F^{*}}{d N}=-\frac{2 V_{m} \gamma_{3}}{a_{3}}+\sum_{i=1}^{I} c_{\beta}^{i} R T \ln \left(\frac{c_{i}^{*}}{c_{i \text { eq }}^{\infty}}\right)
$$

Where $c_{i}^{*}$ is the matrix molar fraction of solute $i$ at the interface, $c_{i \text { eq }}^{\infty}$ is the equilibrium matrix molar fraction as given by the phase diagram (planar interface), $R$ ( $\mathrm{J} \mathrm{mol}^{-}$ $\left.{ }^{1} \mathrm{~K}^{-1}\right)$ is the gas constant and $\gamma_{3}\left(\mathrm{~J} \mathrm{~m}^{-2}\right)$ is the interfacial energy where the surface is perpendicular to the $a_{3}$ axis. Notice that Eq. (4) comes from the following expression of the driving force:

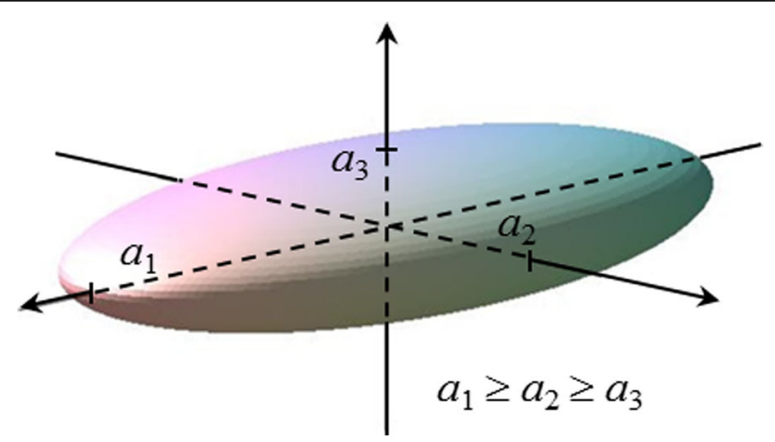

Fig. 1 Ellipsoid with semi-axis of length $a_{1}, a_{2}$ and $a_{3}$ 


$$
\frac{d F^{*}}{d N}=-\frac{2 V_{m} \gamma_{3}}{a_{3}}+\sum_{i=1}^{I} c_{\beta}^{i} R T \ln \left(\frac{a_{i}^{*}}{a_{i \text { eq }}^{\infty}}\right)
$$

where the molar fractions of element $i$ are replaced by the activities of the same element. The two variables are proportional via the chemical thermodynamic factors $\Upsilon$ so that: $a=\Upsilon \cdot c$. In most systems, $\Upsilon$ is nearly a constant when the solute contents are relatively low. In such cases, one can easily justify using Eq. (4) instead of Eq. (5). The term containing $\gamma_{3}$ accounts for the increase in surface free energy when the volume of the precipitate increases. This is known as the Gibbs-Thomson effect. Notice that for a shape invariant particle having full inversion symmetry, Johnson has shown (Johnson, 1965), using the construction given by Wulff, that the ratio $\gamma_{\mathrm{i}} / a_{i}$ is constant in all directions. Eq. (4) can be rewritten as follow:

$$
\frac{d F^{*}}{d N}=R T \sum_{i=1}^{I} c_{\beta}^{i} \cdot \ln \left(\frac{c_{i}^{*}}{c_{i e q}^{*}}\right)
$$

Where:

$$
c_{i e q}^{*}=c_{i e q}^{\infty} \exp \left(\frac{2 V_{m} \gamma_{3}}{I \cdot c_{\beta}^{i} \cdot R T \cdot a_{3}}\right)
$$

The last equation shows that the impact of the Gibbs-Thomson effect is to increase the equilibrium molar fraction of solute in the matrix because of the finite size of the precipitate. If local equilibrium is assumed at the interface, it follows that $c_{i}^{*}=c_{i e q}^{*}$ and the driving force vanishes. According to Eq. (3), the velocity should be equal to zero in this case, unless the interfacial mobility $\rightarrow \infty$ to get a finite migration velocity. But the interfacial mobility cannot be infinite since it is function of temperature and can be expressed as (Christian, 1965):

$$
M=\frac{\Omega}{R T} \exp \left(-\frac{E_{a}}{R T}\right)
$$

where $E_{a}\left(\mathrm{~J} \mathrm{~mol}^{-1}\right)$ is the activation energy for the migration of the interface and $\Omega$ $\left(\mathrm{m}^{4} \mathrm{~mol}^{-1} \mathrm{~s}^{-1}\right)$ is a pre-exponential factor. In fact, the local equilibrium assumption must be understood like $c_{i}^{*} \approx c_{i \text { eq }}^{*}$, but a finite driving force, even small, is always acting when the interface is moving. Such a condition is correct if the precipitate has grown during a time long enough allowing the solute concentration at the interface to get close to its equilibrium value. But when the reaction is just starting, the solute concentration of the matrix at the interface is out of equilibrium. Based on their model of the mixed-mode phase transformations in the solid state, Sietsma and van der Zwaag (Sietsma \& van der Zwaag, 2004) concluded that the initial stage of each phase transformation is interface-controlled in all cases. This implies that the solute concentration in the matrix at the nucleus-matrix interface remains equal to the nominal concentration during the nucleation stage because of the large surface/volume ratio of the nucleus. So if one seeks for a solution which captures the early stage of growth, one has to consider the mobility of the interface in the equations. The problem must then be solved by assuming the mixed-mode regime of growth. 


\section{Mathematical analysis}

The detailed solution of the moving boundary problem of an ellipsoidal precipitate growing with constant eccentricities in the quasi stationary mode has been presented in (Larouche, 2017) for a binary alloy. Without repeating all the details contained in this paper, we will summarize the most important steps of the solution procedure.

The Fick's second law in the quasi-stationary regime can be written as:

$$
D \cdot \nabla^{2} c=\frac{\partial c}{\partial t}=\vec{\nabla} c \cdot \frac{d \vec{r}}{d t}
$$

Where $D$ is the coefficient of diffusion and $\vec{r}$ is the vector position. Assuming a shape conservative growth (eccentricities remain constant) and using the ellipsoidal coordinates, one obtains a general solution:

$$
c-\bar{c}=\left(c^{*}-\bar{c}\right)\left[\frac{F(\xi)}{F\left(\xi_{0}\right)}\right]
$$

where $c^{\prime \prime}$ is the concentration at the interface, $\bar{c}$ is the concentration at infinity, $\xi$ is the ellipsoidal coordinate defined in the reference frame travelling with the moving boundary, $\xi_{0}$ is the ellipsoidal coordinate corresponding to the interface in this frame and $F(\xi)$ is a particular function of $\xi$ expressed as:

$$
F(\xi)=\int_{\infty}^{\xi} \frac{\exp \left(-\frac{k^{2} \xi^{2}}{4 D}\right) d \xi}{\sqrt{\xi^{2}-H_{3}^{2}} \sqrt{\xi^{2}-H_{2}^{2}}}
$$

The variables $\xi, H_{2}$ and $H_{3}$ are related to the static (Eulerian) frame and the shape of the boundary according to the following relationships:

$$
\begin{aligned}
& \xi=\rho / k t^{1 / 2} \\
& H_{2}=\frac{\sqrt{a_{1}^{2}-a_{3}^{2}}}{k t^{1 / 2}} \\
& H_{3}=\frac{\sqrt{a_{1}^{2}-a_{2}^{2}}}{k t^{1 / 2}}
\end{aligned}
$$

Where $t$ is the time and $\rho$ corresponds to an ellipsoidal surface in the Eulerian ellipsoidal system of coordinates. The constant $k$ is called the interface migration coefficient because it was introduced with the travelling set of coordinates. At this stage, there are 3 variables that have to be determined for a given time. These are the size of the precipitate $a_{1}$, the concentration at the interface $c^{\prime \prime}$ and the constant $k$. This constant must be set in order to adapt the velocity of the reference frame $V_{r}$ to the velocity of the interface at the beginning of the mixed-mode regime. Before this time $\left(t_{c}\right)$, the growth is assumed to be $100 \%$ controlled by the interface. The solution is valid only after this time.

Two boundary conditions must be imposed at the interface:

BCAMass conservation across the interface (Stefan condition) 
$\dot{N}=-\int_{S} J \cdot d S=\left(c_{\beta}^{*}-c^{*}\right) \cdot \frac{d V}{d t}$, where $S$ and $V$ are respectively the surface and the volume of the precipitate

BC Zrowth velocity governed by the mobility of the interface as expressed by Eq. (3)

With these 2 boundary conditions, one can determine $a_{1}$ and $c^{*}$ for any time $>t_{c}$, once the constant $k$ has been set. This procedure is sufficient for a binary alloy. But when two solutes and more diffuse during the growth of a precipitate, we have as many Stefan boundary conditions (BC \#1) as there are species involved.

Now, we will rewrite the boundary conditions for the multicomponent alloys, assuming that the partial molar volume of the elements are the same and invariant in both phases. Following the developments detailed in (Larouche, 2017), the BC\#1 condition applied to each solute $i$ can be written as:

$$
\begin{aligned}
\frac{d a_{1}}{d t}= & \frac{D_{i}}{a_{1}^{2} \cdot \sqrt{1-e_{12}^{2}} \sqrt{1-e_{31}^{2}}} \\
& \cdot \frac{\left(c_{i}^{*}-\bar{c}_{i}\right)}{\left(c_{\beta}^{i}-c_{i}^{*}\right)} \frac{\exp \left(-\frac{k^{2} \xi_{0}^{2}}{4 D_{i}}\right)}{\int_{\infty}^{\xi_{0}}\left(\xi^{2}-H_{3}^{2}\right)^{-1 / 2}\left(\xi^{2}-H_{2}^{2}\right)^{-1 / 2} \exp \left(-\frac{k^{2} \xi^{2}}{4 D_{i}}\right) d \xi}
\end{aligned}
$$

where:

$\bar{c}_{i}$ is the nominal molar fraction of solute $i$ in the matrix,

$D_{i}\left(\mathrm{~m}^{2} \mathrm{~s}^{-1}\right)$ is the coefficient of diffusion of solute $i$ in the matrix phase,

$e_{12}$ and $e_{31}$ are the eccentricities of the ellipsoidal precipitate. These are given by:

$$
\begin{aligned}
& e_{12}=\sqrt{1-\left(a_{2} / a_{1}\right)^{2}} \\
& e_{31}=\sqrt{1-\left(a_{3} / a_{1}\right)^{2}}
\end{aligned}
$$

Notice that the boundary condition expressed by Eq. (15) is the same as Eq. (40) in (Larouche, 2017), except that we have now as many conditions to satisfy that there are species condensing onto the precipitate. Inserting Eqs (12), (13) and (14) into (15), one obtains:

$$
\frac{d a_{1}}{d t}=\frac{1}{a_{1}^{2} \cdot k \sqrt{t}} \cdot\left(\frac{\bar{c}_{i}-c_{i}^{*}}{c_{\beta}^{i}-c_{i}^{*}}\right) \cdot \frac{\left(1-e_{12}^{2}\right)^{-1 / 2}\left(1-e_{31}^{2}\right)^{-1 / 2} \cdot D_{i} \exp \left(-\frac{a_{1}^{2}}{4 D_{i} \cdot t}\right)}{\int_{a_{1}}^{\infty}\left(\rho^{2}-e_{12}^{2} a_{1}^{2}\right)^{-1 / 2}\left(\rho^{2}-e_{31}^{2} a_{1}^{2}\right)^{-1 / 2} \exp \left(-\frac{\rho^{2}}{4 D_{i} \cdot t}\right) d \rho}
$$

The other boundary condition to apply at the interface is the one imposed by Eq. (3).

$$
\frac{d a_{1}}{d t}=\frac{M R T}{V_{m}} \sum_{i=1}^{I} c_{\beta}^{i} \cdot \ln \left(\frac{c_{i}^{*}}{c_{i \text { eq }}^{*}}\right)
$$

Now we have to evaluate the constant $k$. The particularity of the mixed-mode model is that it does not start at time 0 . It starts at a time called $t_{c}$, which is the time spent 
during the $100 \%$ interface control regime. At this time, it is expected that the precipitate grows steadily in a quasi-stationary mode, which is characterized by the interface migration coefficient $k$. Figure 2 presents the expected evolution of the interfacial velocity of a precipitate growing in a supersaturated matrix.

Before $t_{c}$, one assumes that the growth rate of a precipitate emerging successfully from the nucleation process is limited by its interfacial mobility. The maximum velocity attainable during this period, called $v_{c}$, is obtained when the full driving force is applied, which occurs when $c_{i}^{*}=\bar{c}_{i}$ in Eq. (19): Therefore, one can write:

$$
v_{c}=\frac{M R T}{V_{m}} \sum_{i=1}^{I} c_{\beta}^{i} \cdot \ln \left(\frac{\bar{c}_{i}}{c_{i \text { eq }}^{*}}\right)
$$

So, at $t=t_{c}$, the size of the precipitate is given by:

$$
a_{c}=v_{c} \cdot t_{c}
$$

Using the procedure followed in (Larouche, 2017), one can show that the velocity of the reference frame following the interface in the mixed mode regime is given by:

$$
V_{r}=\frac{a_{1}}{2 k t^{3 / 2}}
$$

Combining Eqs (21) and Eq. (22), the latter evaluated at $t=t_{c}, a_{1}=a_{c}$ and $V_{r}=\mathrm{v}_{\mathrm{c}}$, on obtains:

$$
k=\frac{1}{2} \sqrt{\frac{v_{c}}{a_{c}}}
$$

Equation (18) must give the same value of $d a_{1} / d t$ for all species involved in the reaction. So, one can establish from this equation a set of $I-1$ equations enforcing the dependency between the flux of solutes:

$$
\text { Fori } i \neq j: \quad f\left(D_{i}\right) \cdot\left(\frac{c_{i}^{*}-\bar{c}_{i}}{c_{\beta}^{i}-c_{i}^{*}}\right)=f\left(D_{j}\right) \cdot\left(\frac{c_{j}^{*}-\bar{c}_{j}}{c_{\beta}^{j}-c_{j}^{*}}\right)
$$

Where:

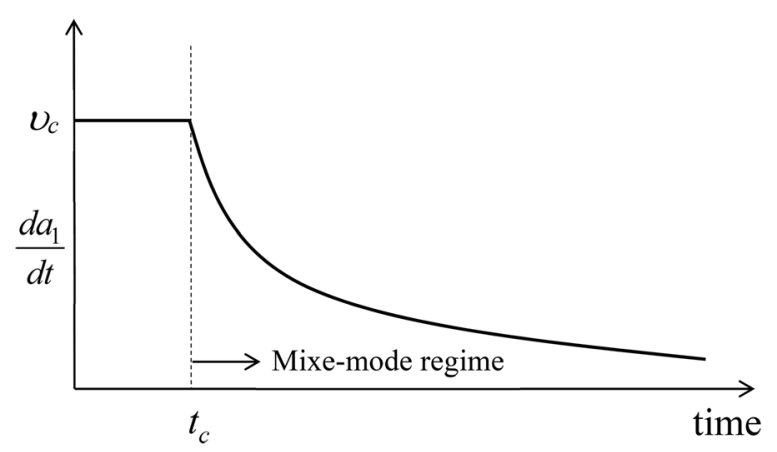

Fig. 2 Expected evolution of the interfacial velocity of a precipitate growing in a supersaturated matrix 


$$
f\left(D_{i}\right)=\frac{D_{i} \exp \left(-\frac{a_{1}^{2}}{4 D_{i} \cdot t}\right)}{\int_{a_{1}}^{\infty}\left(\rho^{2}-e_{12}^{2} a_{1}^{2}\right)^{-1 / 2}\left(\rho^{2}-e_{31}^{2} a_{1}^{2}\right)^{-1 / 2} \exp \left(-\frac{\rho^{2}}{4 D_{i} \cdot t}\right) d \rho}
$$

If the solute $j=1$, is used as the independent specie, which could be anyone of the species involved, the set of equations can be reduced to only 1 equation with one unknown. First, one has to enforce the equality between the velocity of the reference frame, Eq. (22), and the velocity imposed by Eq. (19):

$$
\frac{a_{1}}{2 k t^{3 / 2}}=\frac{M R T}{V_{m}} \sum_{i=1}^{I} c_{\beta}^{i} \cdot \ln \left(\frac{c_{i}^{*}}{c_{i \text { eq }}^{*}}\right)
$$

Isolating $c_{i}^{*}$ from Eq. (24) and inserting the result into Eq. (26), then one obtains:

$$
\frac{a_{1}}{2 k t^{3 / 2}}=\frac{M R T}{V_{m}} \sum_{i=1}^{I} c_{\beta}^{i} \cdot \ln \left(\frac{\bar{c}_{i}+c_{\beta}^{i} \frac{f\left(D_{1}\right)}{f\left(D_{i}\right)} \cdot\left(\frac{c_{1}^{*}-\bar{c}_{1}}{c_{\beta}^{1}-c_{1}^{*}}\right)}{c_{i \text { eq }}^{*}\left[1+\frac{f\left(D_{1}\right)}{f\left(D_{i}\right)} \cdot\left(\frac{c_{1}^{*}-\bar{c}_{1}}{c_{\beta}^{1}-c_{1}^{*}}\right)\right]}\right)
$$

Now, one has to enforce the equality between the velocity of the reference frame, Eq. (22), and the velocity imposed by the Stefan boundary condition, Eq. (18). This gives the following equation:

$$
\frac{\bar{c}_{i}-c_{i}^{*}}{c_{\beta}^{i}-c_{i}^{*}}=\frac{a_{1}^{3} \sqrt{1-e_{12}^{2}} \sqrt{1-e_{31}^{2}}}{2 D_{i} \cdot t} \exp \left(\frac{a_{1}^{2}}{4 D_{i} \cdot t}\right) \int_{a_{1}}^{\infty}\left[\left(\rho^{2}-e_{12}^{2} a_{1}^{2}\right)^{-1 / 2}\left(\rho^{2}-e_{31}^{2} a_{1}^{2}\right)^{-1 / 2} \exp \left(-\frac{\rho^{2}}{4 D_{i} \cdot t}\right)\right] d \rho
$$

The variable $c_{1}^{*}$ can be isolated from Eq. (28) by setting $i=1$. The expression obtained is inserted into Eq. (27) and one obtains one equation with $a_{1}$ as the unknown. The latter is determined at a given time $t$ by solving this equation. Once $a_{1}$ is known, the value of $c_{1}^{*}$ can be retrieved from Eq. (28) and the other $c_{i}^{*}$ from the following equation:

$$
c_{i}^{*}=\left[\bar{c}_{i}+c_{\beta}^{i} \frac{f\left(D_{1}\right)}{f\left(D_{i}\right)} \cdot\left(\frac{c_{1}^{*}-\bar{c}_{1}}{c_{\beta}^{1}-c_{1}^{*}}\right)\right] \cdot\left[1+\frac{f\left(D_{1}\right)}{f\left(D_{i}\right)} \cdot\left(\frac{c_{1}^{*}-\bar{c}_{1}}{c_{\beta}^{1}-c_{1}^{*}}\right)\right]^{-1}
$$

The solution is then completed.

\section{Application of the model}

We will consider the growth of a needle-shape $\beta$ " precipitate in an aluminium matrix having the nominal composition given in Table 1.

The precipitate will grow at a temperature of $453 \mathrm{~K}$ and, at the beginning of the mixed-mode regime, the dimensions of the needle-shape precipitate are assumed to be:

$$
a_{1}=a_{c}=6 \mathrm{~nm}
$$

Table 1 Chemical composition of the alloy used for the mixed-mode growth modelling

\begin{tabular}{llll}
\hline & $\mathrm{Si}$ & $\mathrm{Mg}$ & $\mathrm{Al}$ \\
\hline at.\% & 1.24 & 0.35 & Bal. \\
\hline
\end{tabular}




$$
a_{2}=a_{3}=0.67 \mathrm{~nm}
$$

We will suppose that this size is sufficiently large to ignore the Gibbs-Thomson effect. Therefore the surface energy $\gamma$ will be made equal to 0 . Table 2 gives the equilibrium data calculated with the MatCalc software v. 5.62 (Kozeschnik et al., 2004) using the thermodynamic, mobility diffusion and physical property databases assessed by Povoden-Karadeniz (Povoden-Karadeniz, 2015a; Povoden-Karadeniz, 2015b; Povoden-Karadeniz, 2012).

\section{Results}

Figure 3 presents the evolution of the semi-axis length $a_{1}$, as a function of time, of the $\beta$ " precipitate calculated with two different interfacial mobilities. The two curves merge when the mixed-mode regime becomes essentially diffusion controlled in both cases. The time after which the process may be considered as diffusion controlled depends on the mobility of the interface. Since the mobility is a thermally activated process, one may expect that it will have a strong impact on the growth behavior at low temperatures. The time evolutions of $\mathrm{Mg}$ and $\mathrm{Si}$ concentration at the interface of the matrix are shown in Fig. 4. Both the concentrations of solutes at the interface start at the nominal concentrations and decrease at a rate which depends on the interfacial mobility. For very long times, the concentrations at the interface stabilize at a value which differs from the equilibrium value. For magnesium, the stabilized concentration is above the equilibrium value while for silicon, it is below. It is easy to show that the combined driving force calculated with Eq. (4) is vanishing with time, the negative driving force of silicon cancelling the positive driving force of the other species ( $\mathrm{Mg}$ and $\mathrm{Al}$ ). This result indicates that when the mixed-mode growth is becoming largely diffusion controlled, the total driving force at the interface is getting close to zero. In a multicomponent system, this condition means that the sum of each driving force will be zero, though the interfacial concentration of each species will not be equal to the full equilibrium concentration. The offset obtained between the stabilized concentration and the equilibrium concentration comes from the fact that the quasi stationary growth regime is not equivalent to the full equilibrium situation. In the former case, the nominal concentration still prevails at infinity and consequently the average concentrations remain equal to the nominal concentrations. But in a finite system, the average concentrations gradually change towards their equilibrium values as the growth proceeds. The full equilibrium situation is therefore only possible for a finite system.

Table 2 Equilibrium values calculated at $453 \mathrm{~K}$ and $101 \mathrm{kPa}$ with the nominal composition given in Table 1. The Al matrix and the precipitate $\beta$ " were considered as the only active phases

\begin{tabular}{ll}
\hline Molar fraction of $\mathrm{Mg}$ in the precipitate: $\boldsymbol{c}_{\boldsymbol{\beta}, M g}^{*}$ & 0.4545 \\
Molar fraction of $\mathrm{Si}$ in the precipitate: $\boldsymbol{c}_{\boldsymbol{\beta}, \boldsymbol{S i}}^{*}$ & 0.5105 \\
Molar fraction of $\mathrm{Al}$ in the precipitate: $\boldsymbol{c}_{\boldsymbol{\beta}, \boldsymbol{A l}}^{*}$ & 0.0350 \\
Molar fraction of $\mathrm{Mg}$ in the matrix: $\boldsymbol{c}_{\boldsymbol{e q}, \mathbf{M g}}^{\infty}$ & $7.06 \mathrm{E}-5$ \\
Molar fraction of Si in the matrix: $\boldsymbol{c}_{\boldsymbol{e q}, \boldsymbol{S i}}^{\infty}$ & $8.61 \mathrm{E}-3$ \\
Coefficient of diffusion of $\mathrm{Mg}$ in the matrix $\left(\mathrm{m}^{2} / \mathrm{s}\right)$ & $2.01 \mathrm{E}-19$ \\
Coefficient of diffusion of Si in the matrix $\left(\mathrm{m}^{2} / \mathrm{s}\right)$ & $1.35 \mathrm{E}-19$ \\
Molar volume of the precipitate $\left(\mathrm{m}^{3} / \mathrm{mol}^{\prime}\right)$ & $1.048 \mathrm{E}-5$ \\
\hline
\end{tabular}




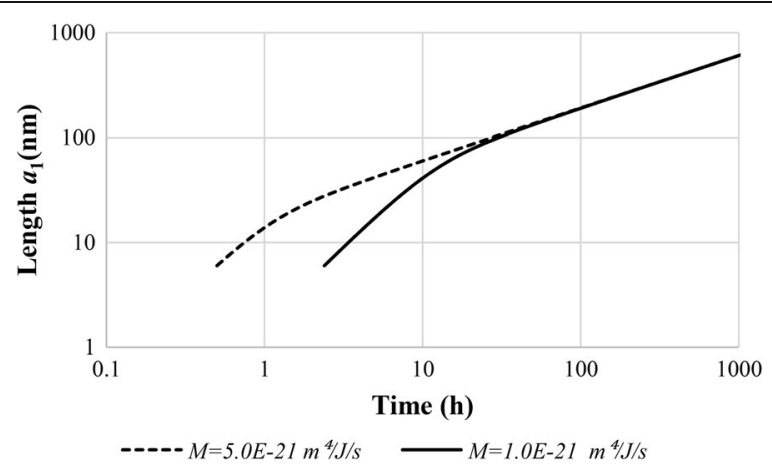

Fig. 3 Time evolution of the semi-axis length $a_{1}$ calculated using 2 different interfacial mobilities

\section{Discussion}

For the first time, an analytical solution is provided for the mixed-mode growth of a multicomponent precipitate, the latter growing with the simultaneous diffusion of several species in the matrix. In the example described above, 3 species were involved in the formation of the precipitate. The method can include as much species as wanted since it is always possible to reduce the number of equations to 1 , with only 1 unknown to find. This unknown is the size of the precipitate at a given time. Once this variable is determined, the concentrations of all species at the interface can be calculated easily. The method can therefore be applied for the growth of any type of particle, under the condition that its composition is known and invariant. The applicability of the solution is limited to situations where each precipitate grows in a relatively large matrix since the solution is exact for one precipitate growing in an infinite matrix. This is the same limitation which applies with the popular Zener stationary approximate solution. However, the mixed-mode growth solution takes into account the moving boundary problem under the assumption of a quasi-stationary growth, unlike the Zener stationary approximate solution, which ignores completely the term $\partial c / \partial t$ in the mass conservation equation.

The time evolution of the concentrations at the matrix interface $\left(c_{i}^{*}\right)$ is certainly the most interesting result obtained as it can be considered as characteristic of the mixed-mode growth. During the early stages of growth, the interface concentrations are equal to the nominal concentrations. After, they vary gradually with time toward their stabilized value at a rate controlled by the interfacial mobility. The offset between the final and equilibrium concentrations is produced by the difference existing between the coefficients of diffusion of the 2 solutes. Indeed, if one makes the calculations using the same coefficients of diffusion for the solutes, then one obtains a perfect match between the final and equilibrium values. Unlike the case where equilibrium is assumed at the interface, the time evolution of $c_{i}^{*}$ is free of the drastic change occurring at the beginning of the growth. This gives a more realistic solution since the interfacial mobility is surely not infinite.

Diffusional growth of multicomponent precipitates can be simulated with the commercial application software DICTRA, the latter being linked with the thermodynamic software package Thermo-Calc (Andersson et al., 2002) for the calculation of equilibrium variables. DICTRA provides numerical solutions of the multicomponent diffusion 

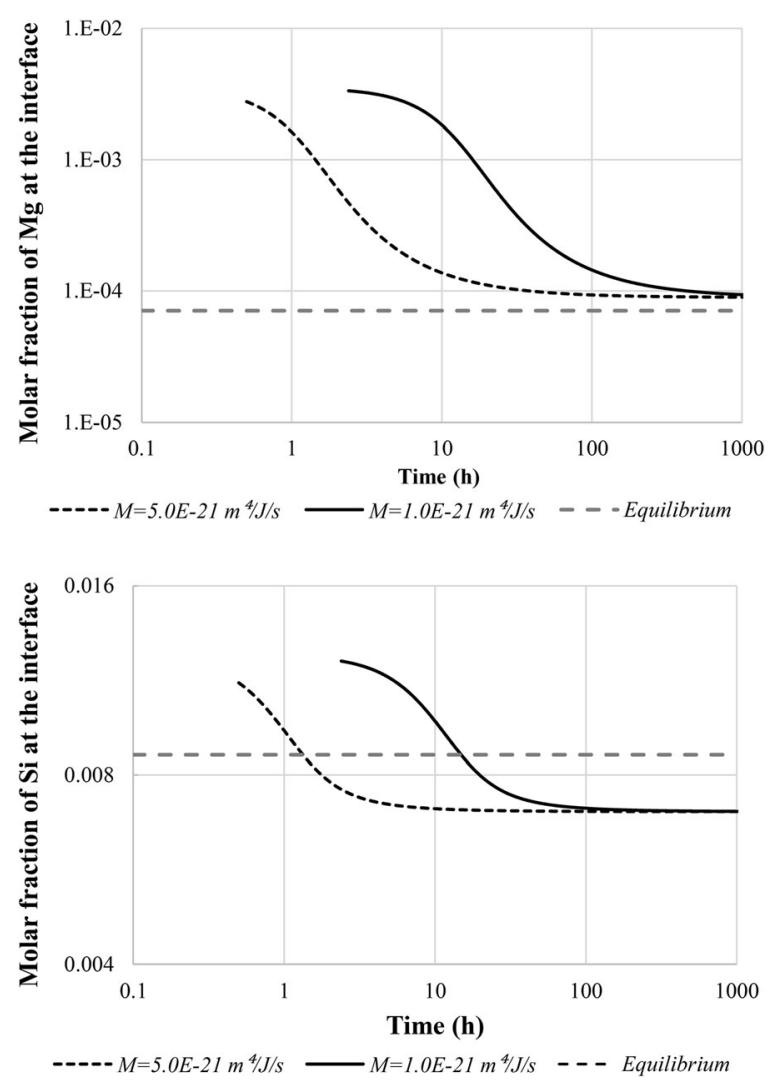

Fig. 4 Evolution of the solute molar fractions, as a function of time, at the interface of a needle-shape $\beta^{\prime \prime}$ precipitate growing in an aluminium matrix as calculated by the mixed-mode model

equations in one dimension (planar, cylindrical or spherical) and perform a mass balance of diffusional flux across the interface to calculate the velocity of a moving boundary. The reader interested in the mathematics behind DICTRA should consult reference (Ågren, 1982) for the details relevant to the moving boundary problem. The main assumption used by DICTRA is that equilibrium is achieved at the interface, which means that the concentrations on both sides of the interface are ruled by the phase diagram. This assumption is called local equilibrium. Few contributions are available in the literature where DICTRA was used to simulate the growth of precipitates during the early stage of precipitation. The most prominent examples were proposed for the growth of MxCy carbides in Fe rich matrix. Where M can be Fe or $\mathrm{Cr}$ (Chen et al., 2008; Schneider \& Inden, 2005; Ghosh \& Olson, 2002). At the knowledge of the authors, no examples have been published describing the early stage of precipitation in aluminium alloys with DICTRA. A one-to-one comparison between our model and DICTRA cannot be made since the former provides a solution for an infinite system while the latter provides a solution for a finite system. But if the finite system is made large enough, the solutions can be compared at least during the early stage of growth. A DICTRA model simulating the growth of a spherical $\mathrm{Mg}_{2} \mathrm{Si}$ particle in a rich Aluminium matrix having the composition given in Table 1 was built for comparison purposes. Since DICTRA does not simulate the growth of needle-like precipitates, one has to set a mixed-mode calculation for a spherical-type of $\mathrm{Mg}_{2} \mathrm{Si}$ precipitate using the same value for the different parameters. The equilibrium values used in the 
mixed-mode model were calculated with Thermo-Calc 2016a and the TTAl7 database (TTAL7, 2010). The TTAl7 database was also used in the DICTRA simulation. Constant coefficients of diffusion were set in the DICTRA macro using the command "ENTER_MOBILITY_ESTIMATE". The coefficient of diffusions and equilibrium concentrations were therefore the same in the mixed-mode calculation and the DICTRA simulation. Table 3 summarizes the data used for the DICTRA and the mixed-mode calculations.

Figure 5 presents the evolution of the solute interfacial concentrations as calculated by the two models. According to the DICTRA simulation, the interfacial concentration of silicon varies between 0.831 at\% and 1.07 at\% during the first $70 \mathrm{~s}$ and then stabilises at 0.980 at $\%$ for a while before increasing steadily toward the full equilibrium concentration (1.07 at\%). For $\mathrm{Mg}$, the DICTRA simulation predicts a very rapid stabilization of the interfacial concentration to the expected full equilibrium concentration. The mass balance is made possible in this example by the interfacial concentration of $\mathrm{Si}$, which deviates from the full equilibrium concentration at the beginning of the reaction until the finite size of the system allows attaining the full equilibrium situation. What is interesting to notice is that, after the short transient period of $70 \mathrm{~s}$, the interfacial concentration of Si predicted by DICTRA remains constant for approximately one hour at a value which differs by only $0.013 \%$ of the final equilibrium interfacial concentration of Si predicted by the mixed-mode model ( $0.980 \%$ versus $0.993 \%)$. During this hour of growth, the size of the precipitate remains small in comparison to the size of the system, so one can say that the growth regime during this period is close to the quasi-stationary growth of a precipitate in an infinite system. The only difference is that the mixed-mode model assumes a finite interfacial mobility instead of an infinite one, so the time to reach the stabilized concentration is much longer for the mixed-mode than it is for the diffusion control mode, the latter being theoretically stabilized at time zero. But DICTRA needed 40 time steps to reach this quasi stabilized regime since the process has to search for starting values, the latter depending on the phase diagram. This search of starting values is not always successful in DICTRA simulations and, when successful, the evolution of the interfacial concentration can be somewhat erratic during the first steps since they are influenced by the setting values used in the numerical computation. With the mixed-mode model, these stabilized concentrations can be

Table 3 Equilibrium values calculated at $453 \mathrm{~K}$ and $101 \mathrm{kPa}$ with the nominal composition given in Table 1. The Al matrix and the precipitate $\beta-\mathrm{Mg}_{2} \mathrm{Si}$ were considered as the only active phases

\begin{tabular}{|c|c|}
\hline Molar fraction of $\mathrm{Mg}$ in the precipitate: $\boldsymbol{c}_{\beta, M g}^{*}$ & 0.6667 \\
\hline Molar fraction of Si in the precipitate: $c_{\beta, S i}^{*}$ & 0.3333 \\
\hline Molar fraction of $\mathrm{Mg}$ in the matrix: $\mathbf{C}_{\mathbf{e q}, \mathbf{M g}}^{\infty}$ & $2.14 \mathrm{E}-6$ \\
\hline Molar fraction of Si in the matrix: $\boldsymbol{c}_{e q, S i}^{\infty}$ & $1.07 \mathrm{E}-2$ \\
\hline Coefficient of diffusion of $\mathrm{Mg}$ in the matrix $\left(\mathrm{m}^{2} / \mathrm{s}\right)$ & 2.01E-19 \\
\hline Coefficient of diffusion of $\mathrm{Si}$ in the matrix $\left(\mathrm{m}^{2} / \mathrm{s}\right)$ & 1.35E-19 \\
\hline Molar volume of the precipitate $\left(\mathrm{m}^{3} / \mathrm{mol}\right)$ & $1.000 \mathrm{E}-5$ \\
\hline Radius of precipitate at the start of the mixed-mode regime: $a_{c}(\mathrm{~nm})$ & 0.4 \\
\hline Radius of precipitate at the start of the DICTRA simulation (nm) & 0.4 \\
\hline Radius of the system in the DICTRA simulation (nm) & 50 \\
\hline Surface energy: $ү$ : & 0 \\
\hline
\end{tabular}



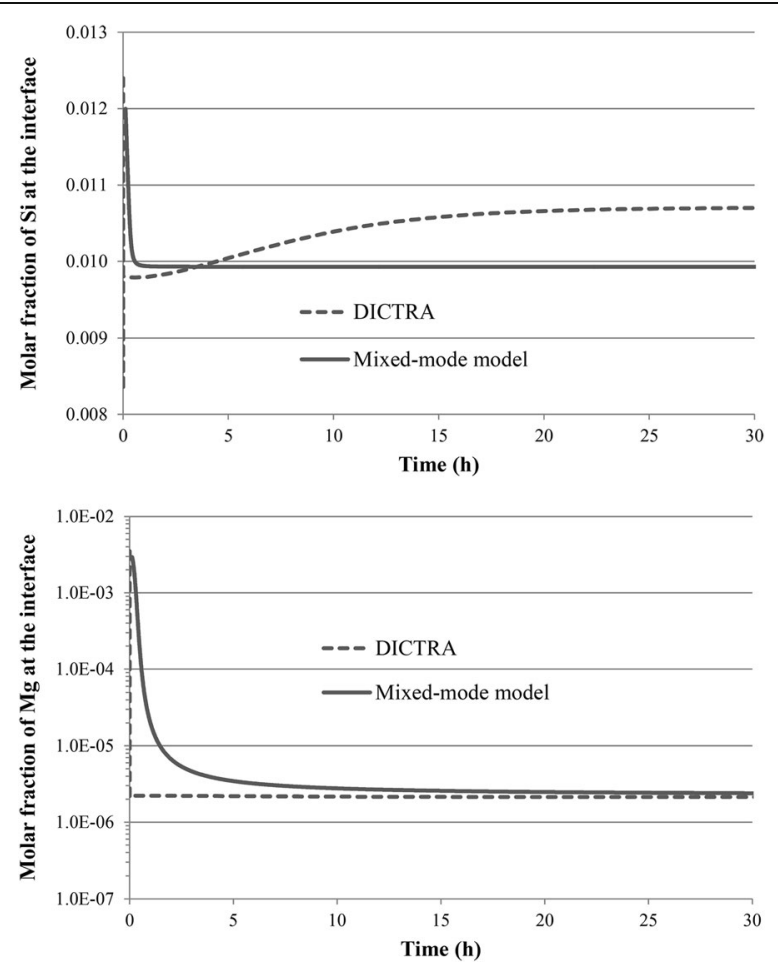

Fig. 5 Evolution of the solute molar fractions at the interface as calculated by the two models

calculated in a one step procedure by setting a very long time. This result indicates that interfacial concentrations are not governed by alternative tie-lines, but only by the coefficients of diffusion and the full equilibrium concentrations. No doubt that the robustness of an application tool like DICTRA to simulate moving boundary problems would be enhanced by using a boundary condition involving the mobility of the interface as expressed by Eq. (3), instead of the local equilibrium assumption. If the interfacial mobility is unknown, it would always be possible to find the diffusion controlled solution by using the interfacial mobility as a penalty variable.

\section{Conclusion}

An exact analytical solution of the mixed-mode growth of a multicomponent ellipsoidal precipitate having constant eccentricities was developed for the quasi stationary regime. The solution was applied to simulate the growth of a needle shape (prolate spheroid) $\mathrm{Al}-\mathrm{Mg}-\mathrm{Si}$ compound and a spherical $\mathrm{Mg}_{2} \mathrm{Si}$ compound growing in their aluminium based matrix. A comparison made with the diffusion-controlled simulation software DICTRA showed that the solute concentrations at the interface in the early stages of the reaction can be found irrespective of local equilibrium considerations. The variables having a significant effect are the full equilibrium concentrations, the nominal composition and the coefficients of diffusion. With the mixed-mode growth approach, multicomponent mapping of the phase diagram, allowing the determination of alternative tie-lines, is not necessary to solve the theoretical model.

\section{Acknowledgements}

The authors thanks Martin Fortier of RioTinto and Denis Massinon of Linamar Montupet Light Metal Casting for their support. 


\section{Funding}

This work was supported financially by the Natural Sciences and Engineering Research Council of Canada (Grant RDCPJ 468550-14) and the industrial partners RioTinto and Montupet.

\section{Authors' contributions}

TN and DL made the theoretical developments and calculations presented in this paper. RM and FB analyzed the results and critically evaluated the relevance of the approach for the simulation of precipitation kinetics in multicomponent alloys. All authors read and approved the final manuscript.

\section{Competing interests}

The authors declare that they have no competing interests.

\section{Author details}

'Department of Mining, Metallurgy and Materials Engineering, Aluminum Research Center - REGAL, Laval University, 1065, Ave de la Médecine, Quebec G1V OA6, Canada. ${ }^{2}$ Linamar Montupet Light Metal Casting, 32233 West Eight Mile Road, Livonia, MI 48152, USA. ${ }^{3}$ RioTinto, 1955 Mellon Blvd, Jonquière, Quebec G7S 4K8, Canada.

Received: 2 February 2018 Accepted: 17 May 2018

Published online: 23 May 2018

References

HI Aaronson, M Enomoto, JK Lee, Mechanisms of Diffusional Phase Transformations in Metals and Alloys (Taylor \& Francis, Boca Raton, 2010)

J Ågren, Numerical treatment of diffusional reactions in multicomponent alloys. J. Phys. Chem. Solids 43(4), 385-391 (1982)

J-O Andersson, T Helander, L Hoglund, P Shi, B Sundman, Thermo-Calc \& DICTRA, computational tools for materials science. Calphad 26(2), 273-312 (2002)

Q Chen, J Jeppsson, J Ågren, Analytical treatment of diffusion during precipitate growth in multicomponent systems. Acta Materi. 56, 1890-1896 (2008)

JW Christian, The Theory of Transformations in Metals and Alloys; an Advanced Textbook in Physical Metallurgy (Pergamon Press, Oxford, 1965)

G Ghosh, G Olson, Precipitation of paraequilibrium cementite: Experiments, and thermodynamic and kinetic modeling. Acta Mater. 50(8), 2099-2119 (2002)

FS Ham, Shape-preserving solutions of the time-dependent diffusion equation. Q. Appl. Math. 17(2), 137-145 (1959)

M Hillert, Phase Equilibria, Phase Diagrams and Phase Transformations: Their Thermodynamic Basis, 2nd edn. (Cambridge University Press, Cambridge, 2008)

G Horvay, JW Cahn, Dendritic and spheroidal growth. Acta Metall. 9(7), 695-705 (1961)

CA Johnson, Generalization of the Gibbs-Thomson equation. Surf. Sci. 3(5), 429-444 (1965)

JS Kirkaldy, Diffusion in multicomponent metallic systems .2. Solutions for 2-phase systems with applications to transformations in steel. Can. J. Phys. 36(7), 907-916 (1958)

E Kozeschnik, Modeling Solid-State Precipitation (Momentum Press, New York, 2013a)

E Kozeschnik, Modeling Solid-State Precipitation, vol 131 (Momentum Press, New York, 2013b)

E Kozeschnik, J Svoboda, P Fratzl, FD Fischer, Modelling of kinetics in multi-component multi-phase systems with spherical precipitates: II: Numerical solution and application. Mater. Sci. Eng. A 385, 157-165 (2004)

D Larouche, Mixed mode growth of an ellipsoidal precipitate: Analytical solution for shape preserving growth in the quasi-stationary regime. Acta Mater. 123, 188-196 (2017)

E. Povoden-Karadeniz. Physical Properties Data from MatCalc Database 'mc_al.pdb' Version 1.019, (2012).

E. Povoden-Karadeniz. Thermodynamic Data from MatCalc Database 'mc_al.tdb',Version 2.030, (2015a).

E. Povoden-Karadeniz. Mobility Diffusion Data from MatCalc Database 'mc_al.ddb' Version 2.006, (2015b).

A Schneider, G Inden, Simulation of the kinetics of precipitation reactions in ferritic steels. Acta Mater. 53(2), 519-531 (2005)

J Sietsma, S van der Zwaag, A concise model for mixed-mode phase transformations in the solid state. Acta Mater. 52(14), 4143-4152 (2004)

TTAL7, TT Al-Based Alloys Database, Version 7.0 (ThermoTech Ltd., Surrey Technology Center, Guildford, 2010)

C Zener, Theory of growth of spherical precipitates from solid solution. J. Appl. Phys. 20(10), 950-953 (1949) 